\title{
Visual processing of rotary motion
}

\author{
P. WERKHOVEN and J. J. KOENDERINK \\ Utrecht University, Utrecht, The Netherlands
}

\begin{abstract}
Local descriptions of velocity fields (e.g., rotation, divergence, and deformation) contain a wealth of information for form perception and ego motion. In spite of this, human psychophysical performance in estimating these entities has not yet been thoroughly examined. In this paper, we report on the visual discrimination of rotary motion. A sequence of image frames is used to elicit an apparent rotation of an annulus, composed of dots in the frontoparallel plane, around a fixation spot at the center of the annulus. Differential angular velocity thresholds are measured as a function of the angular velocity, the diameter of the annulus, the number of dots, the display time per frame, and the number of frames. The results show a U-shaped dependence of angular velocity discrimination on spatial scale, with minimal Weber fractions of $7 \%$. Experiments with a scatter in the distance of the individual dots to the center of rotation demonstrate that angular velocity cannot be assessed directly; perceived angular velocity depends strongly on the distance of the dots relative to the center of rotation. We suggest that the estimation of rotary motion is mediated by local estimations of linear velocity.
\end{abstract}

Early investigators have noted that deformations of the retinal image (optical flow) due to relative motion between observers and environmental objects provide a rich source of information about the three dimensional spatial layout (Mach, 1886/1962; Helmholtz, 1925). Optical flow fields have been shown to be relevant to the ability of aircraft pilots (Gibson, Olum, \& Rosenblatt, 1958) to estimate their orientation in space. It is also relevant to the steering task of automobile drivers (Riemersma, 1981).

A major objective of study has been to determine the role of the velocity of optical flow as the carrier of information. Gordon (1965) gave a mathematical analysis of the global instantaneous velocity and acceleration fields of optical flow in the context of a dynamic three-dimensional (3-D) environment. An extensive literature has evolved on the physiological mechanisms and models (Horn \& Schunck, 1981; Marr \& Ullman, 1981; Reichardt, 1987; van Santen \& Sperling, 1985) needed to code instantaneous velocity. Human psychophysical performance was measured (Anstis, 1986; De Bruyn \& Orban, 1988; McKee, 1981; McKee \& Welch, 1985; Moulden \& Beggs, 1986; Nakayama, 1985a; van Doorn \& Koenderink, 1982a, 1982b) for tasks such as velocity discrimination, detection, and so forth, to collect experimental evidence for these models.

However, an important step forward was made by Koenderink and van Doorn $(1975,1976)$, who pointed

This work was supported by US Air Force Office of Scientific Research Grant 87-0380 and the Netherlands Organization for Scientific Research (NWO). The preparation of this manuscript was partially supported by USAF Life Science Directorate Visual Information Processing Grant 88-0140. Correspondence should be addressed to Peter Werkhoven, Human Information Processing Laboratory, Department of Psychology and Center for Neural Science, New York University, 6 Washington PI., Rm. 980 E, New York, NY 10003. out that information about 3-D shape and 3-D motion is carried locally by the spatial variation of the instantaneous velocity field (motion parallax). For example, they have shown that in the immediate neighborhood of a visual direction, the motion parallax field can be uniquely decomposed into three linear transformations (motion parallax components): a rotation, a similarity, and a deformation. Each of the elementary transformations is determined by first-order differential invariants of the velocity field (curl, divergence, and shear, respectively) with simple geometrical interpretations. These motion parallax components form important entities that characterize local properties of the motion parallax field in a coordinatefree manner and are directly related to 3-D surface orientation and ego motion.

Longuet-Higgens and Prazdny (1980) have shown that it is theoretically possible to derive self-motion from the retinal velocity field, providing that the motion parallax components are available. Several other algorithms have been designed to estimate environmental geometry and ego motion from motion parallax (Hoffman, 1982; Rieger, 1983; Waxman, Kamar-Parsi, \& Subbarao, 1987), with rigidity assumed and even higher order derivatives of velocity taken as input. Recently, Koenderink and van Doorn (1986) designed an algorithm that estimates 3-D motion and senses spatial orientation of nonrigid polyhedral objects, with local motion parallax components taken as input data.

Since the importance of the local motion parallax structure for visual systems interacting with a dynamic environment has been established (Rogers \& Graham, 1979; Warren, Morris, \& Kalish, 1988), one may ask what visual neural mechanisms actually evolved to code the local structure of motion parallax. It is mostly assumed that the estimation of motion parallax components is the result of a weighted integration of the velocity field (Nakayama, 
1985b; Saito et al., 1986). Nakayama and Loomis (1974) argued for neural structures (convexity cells) that code higher order variables of the optical flow pattern and are both efficient in coding the flow pattern and highly informative about the organization of the surrounding environment with respect to depth. Furthermore, psychophysical experiments by Regan (1986) showed that specific local structures in velocity fields are processed by neural structures that are distinct from elements sensitive to local linear velocities. Moreover, Tanaka, Fukada, and Saito (1989) and Tanaka and Saito (1989) found neurophysiological evidence for mechanisms (receptive fields) that are tuned to both curl and motion in depth. They speculate that these mechanisms can be constructed simply by an integrative convergence of linear motion, forming a hard-wired weighted integration of the velocity field. In conclusion, emerging evidence favors the existence of neural structures, tuned to motion parallax components, that integrate local velocity estimates. However, Nakayama (1985b) discusses three "biological hardware"' limitations imposed on a weighted integration of the velocity field: spatial bandwidth, temporal bandwidth, and the Weber law for differential velocity detection. Therefore, one may expect that human performance of extracting motion parallax components is limited by the properties of detectors of linear motion.

Although extensive literature exists on detection, discrimination, and possible implementation of linear motion detection (for a review, see Nakayama, 1985b), systematic experimental data on coding and the ability to discriminate motion parallax components are, at most, scanty. Such data would be useful to relate the processing of motion parallax to linear motion detection (as a possible input stage) and perhaps to visually guided motor control and to 3-D shape from motion perception.

This paper addresses the processing of the solenoidal component of motion parallax (vorticity) that describes rigid rotation in the frontal plane. We describe four related experiments on the angular velocity discrimination of revolving annuli composed of dots. These experiments independently support the hypothesis that the estimation of angular velocity is mediated by local linear velocity estimations that are fed into higher order routines to code rotation. In Experiment 1, we search for temporal characteristics of the discrimination mechanism. In Experiment 2, we infer properties of the discrimination mechanism by varying the number of dots in a revolving annulus and by the spatial distribution of the dots. In this section, our hypothesis will be elaborated and tested. Experiments 3 and 4, in which spatial scale and angular velocity are varied, provide further support for our hypothesis. We find both scale invariance for a wide range of spatial scales and a U-shaped dependence of discrimination thresholds for angular velocity, with optimal thresholds and range boundaries close to those found for linear motion perception.

\section{GENERAL METHOD}

To examine the psychophysical performance of subjects in discriminating angular velocity, we used stimuli that consisted of an annulus composed of dots revolving around an axis perpendicular through the center of a screen.

\section{Subjects}

Two subjects participated in the experiments (a student, R.O., and the author, P.W.). R.O. is myopic (-2.5 D) and P.W. is emmetropic. Both subjects are experienced observers in psychophysical experiments related to motion perception and are familiar with the objectives of the experiments. In none of the experiments was a marked difference between the subjects observed.

\section{Apparatus}

The stimuli were generated on an Atari 1040 ST computer. An Atari SM125 high resolution $70 \mathrm{~Hz}$ white phosphor monochrome monitor was used (luminance $71 \mathrm{~cd} / \mathrm{m}^{2}$ ). The phosphor decay rate was approximately $0.3 \mathrm{msec}$, which made a spatial blur cue to motion unlikely. The monitor dimensions are $13.6 \times 21.8 \mathrm{~cm}$ $(400 \times 640$ pixels $)$.

\section{Stimulus}

The stimulus used in the experiments consists of a series of $N_{f}$ image frames, displayed contiguous in time ( $\tau$ msec each). The image frame time $\tau$ could be varied in steps of $14.3 \mathrm{msec}$. Thus, the stimulus duration, $T_{s}$, was $T_{s}=N_{f} \tau$ msec. Each image frame was a snapshot of a revolving annulus composed of $N_{d}$ dots with positions $\left(\rho_{i}, \phi_{i}\right)$ in polar coordinates $\left(i=1 \ldots N_{d}\right)$. The dots were regularly distributed in angle $(\phi): \phi_{i}-\phi_{i-1}=2 \pi / N_{d}$. The dots had a distance, $\rho_{i}$, relative to the fixation mark (also a dot) in the center of the screen (Figure 1). The eccentricity, $\rho_{i}$, of the individual dots of an annulus was uniformly distributed within an interval $\left[(1-\epsilon) \rho_{0}\right.$ $\left.\ldots(1+\epsilon) \rho_{0}\right]$, where $\rho_{0}$ is the mean radius.

Hence, the dots had an eccentricity distribution with a standard deviation $\sigma_{0}=\epsilon \rho_{0} / \sqrt{3}$. The annulus was rigidly rotated in the frontal plane around the fixation mark over an angle $(\Omega \tau)$ from one image frame to the next, where $\Omega$ represents the angular velocity of the annulus in $\mathrm{deg} / \mathrm{sec}$. The dots were drawn in black on a white background, so that the visual system adapted to a well-defined luminance level (luminance $71 \mathrm{~cd} / \mathrm{m}^{2}$ ).

The retinal spatial dimensions of the stimulus depend on the viewing distance $D$, which was $100 \mathrm{~cm}$ except for Experiment 3, where the viewing distance was varied between 7 and $1,200 \mathrm{~cm}$. The dots had a size of $0.17 \mathrm{~cm}(5.8 \mathrm{arcmin}$ at $D=100 \mathrm{~cm})$ in all experiments except for Experiment 3, where the size was $0.31 \mathrm{~cm}(10.7$ arcmin at $D=100 \mathrm{~cm})$. The mean radius $\left(\rho_{0}\right)$ was $3 \mathrm{~cm}\left(1.72^{\circ}\right.$ at $D=100 \mathrm{~cm}$ ) in all experiments.

The parameters $\tau, N_{f}, N_{d}, \epsilon$, and $D$ were varied or kept constant in different experiments, as listed in Table 1. Should the choice of parameters for specific experiments require comments or argumentation, they will be given in the sections describing the specific experiments.

\section{Procedure}

Discrimination thresholds were determined in a session in which a subject binocularly viewed 100 pairs of revolving annuli. One pair consisted of a "reference" annulus, revolving with a reference angular velocity, $\Omega_{\text {ref }}$, and a "test" annulus, revolving with a test angular velocity, $\Omega_{\text {test }}$, separated in time $0.25 \mathrm{sec}$ by a blank screen with only a fixation mark. The order of presentation of the reference and test annulus, in one pair, was randomized. In order to avoid having end positions of dots as cues for the discrimination 

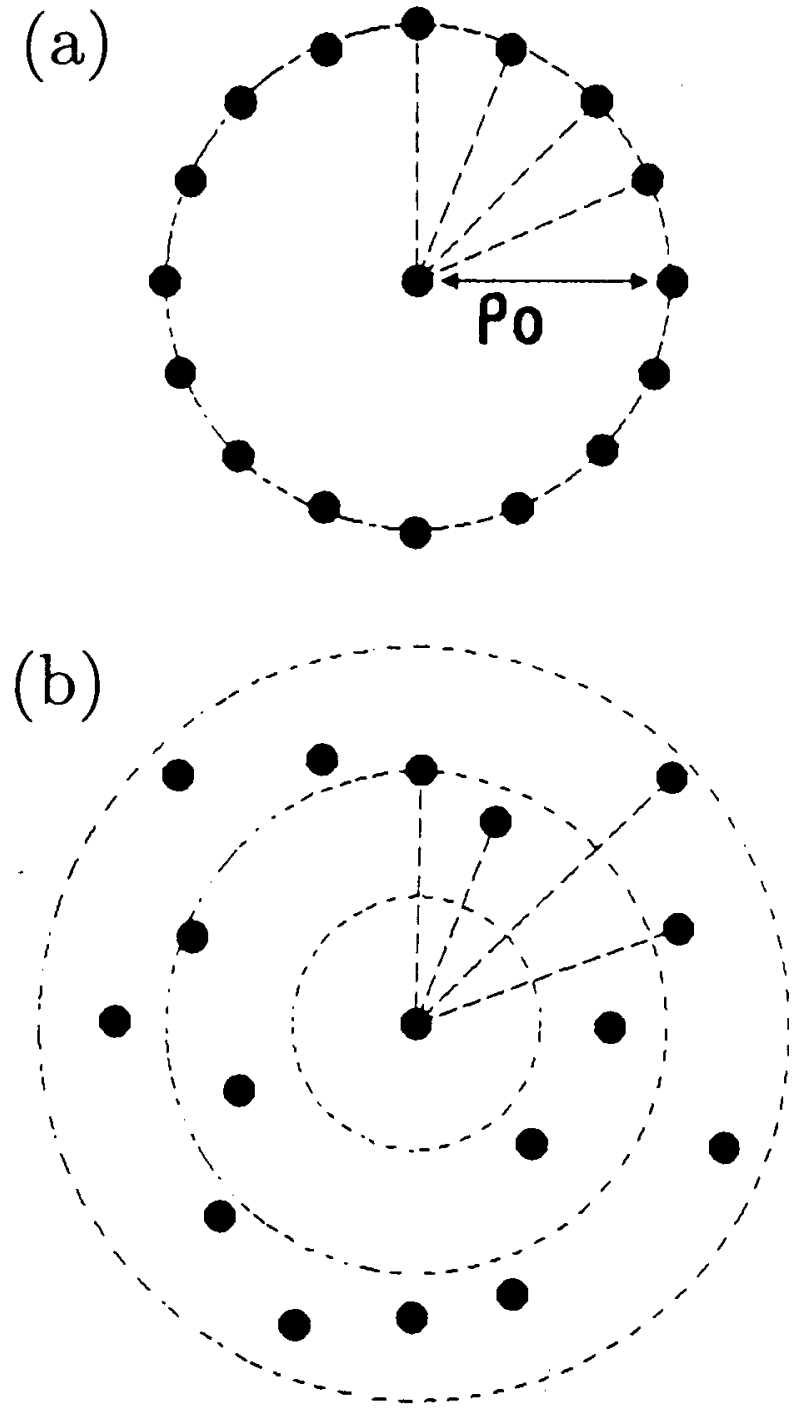

Figure 1. Examples of the arrangement of the dots in an image frame for $N_{d}=16$ are sketched for two situations: (1) the eccentricities $\rho_{i}$ of the dots to the center of rotation (fixation dot in the center) are constant $\left(\epsilon=0\right.$ and thus $\left.\rho_{i}=\rho_{0}\right)$, and (2) the eccentricities $\rho_{i}$ of the dots are distributed $(\epsilon=0.5)$ around $\rho_{0}$ with a standard deviation $\sigma_{\rho}=\rho_{0} / 2 \sqrt{3}$.

of angular velocity, the initial global orientation and the direction of rotation (clockwise, counterclockwise) were also randomized. The task of the subject was to report which of the two annuli (reference or test) in a pair revolved with the highest angular velocity.

We used an adaptive psychometric procedure to estimate two parameters of the psychometric function that describe the psycho- physical performance of the subject: the angular velocity of the test annulus that is perceptually equal to the angular velocity of the reference annulus (i.e., the point of subjective equality $\Omega_{\text {pse }}$ and the threshold angular velocity $\delta \Omega$ ). In general, if the spatiotemporal parameters of the test and reference annulus are identical, $\boldsymbol{\Omega}_{\mathrm{pse}}$ should be equal to $\Omega_{\text {rer. }}$. The discrimination threshold $W=\delta \Omega / \Omega_{\text {ref }}$ is defined as the relative difference between reference angular velocity, $\Omega_{\text {ref, }}$ and test angular velocity, $\Omega_{\text {ref }}+\delta \Omega$, at which the subject is correct in $84 \%$ of the presentations.

The psychometric function is modeled by an error function; the probability $(P)$ that a subject judges the angular velocity of the test annulus $\left(\Omega_{\text {test }}\right)$ to be higher than the angular velocity of the reference annulus $\left(\Omega_{\text {ref }}\right)$ is:

$$
P\left(\Omega_{\text {test }} ; \Omega_{\text {ref }}\right)=\operatorname{Erf}\left(\frac{\Omega_{\text {test }}-\Omega_{\text {ref }}}{\delta \Omega}\right) .
$$

After each trial in a session, maximum likelihood estimates of $\mathbf{\Omega}_{\text {pse }}$ and $\delta \Omega$ were calculated. A next trial was placed at the current most probable estimate of $\Omega_{\text {ref }}+\delta \Omega$ or $\Omega_{\text {ref }}-\delta \Omega$ (with equal probability). It should be noted that $\delta \Omega$ corresponds with the width of the Gaussian distribution that describes the probability distribution of the internal parameter that represents the angular velocity of a revolving annulus.

For the experiments presented in this paper, the measured $\Omega_{\text {pse }}$ were generally distributed with a mean equal to $\Omega_{\text {ref }}$ and a relative standard deviation of approximately $2 \%$. The value $\Omega_{\text {pse }}$ and threshold $\delta \Omega$ were determined four times for every data point for Experiments 1, 3, and 4 and eight times for Experiment 2. It should be noted that the subjects practiced the discrimination task for about 8 sessions or until their performance was shown to be stable. After this training session, we found no marked differences between thresholds measured before and thresholds measured after the experimental sessions. The subjects received feedback in all sessions.

\section{Notation}

In the following sections, discrimination thresholds $W$ will first be given for Subject P.W., followed by second thresholds between brackets [_\%] for Subject R.O.

\section{EXPERIMENT 1 Temporal Characteristics}

Two temporal parameters that can affect discrimination thresholds of angular velocity and show temporal characteristics of the discrimination mechanism are the presentation time $(\tau)$ of one image frame and the total duration, $T_{s}$, of the series of $N_{f}$ image frames $\left(N_{f} \tau\right)$.

\section{Method}

Subjects viewed 8 dots regularly arranged on a circle, revolving at an average angular velocity of $80 \mathrm{deg} / \mathrm{sec}$. Due to the discrete character of apparent motion displays and the arrangement of the dots on a regular polygon, the range within which frame time $\tau$ could be varied without aliasing effects was limited. The direction of rotation is ambiguous at an angular velocity for which the displacement of the dots from one frame to the next is equal to half

Table 1

Parameters as Used in Different Experiments

\begin{tabular}{clllllll}
\hline Experiment & \multicolumn{1}{c}{$N_{d}$} & \multicolumn{1}{c}{$\epsilon$} & \multicolumn{1}{c}{$\tau(\mathrm{msec})$} & \multicolumn{2}{c}{$N_{f}$} & $\mathbf{\Omega}_{\text {ref }}(\mathrm{deg} / \mathrm{sec})$ & $D(\mathrm{~cm})$ \\
\hline 1 & 8 & & 0 & $14 \ldots 114$ & $2 \ldots 64$ & 80 & 100 \\
2 & $1 \ldots 32$ & $0 / 0.5$ & 43 & 8 & 80 & 100 \\
3 & 8 & & 0 & 43 & 8 & 80 & $7 \ldots 1200$ \\
4 & 8 & 0 & 14 & 24 & $10 \ldots 320$ & 100 \\
\hline
\end{tabular}


of the separation of two neighbor dots in one frame. One may expect a gradual change in the probability to perceive reversed rotation around this point. Experiments, on which we will report in detail in another paper, showed that reversed rotation does not occur up to an angular velocity of $84,140,300$, and $840 \mathrm{deg} / \mathrm{sec}$ for frame times $\tau=229,114,43$, and $14 \mathrm{msec}$, respectively. In order to avoid aliasing effects in our experiments, we varied $\tau$ between 14 and $114 \mathrm{msec}$. For a certain value of $r$ within this range, several numbers of frames were used, with $\tau$ a power of two. The stimulus duration $\left(T_{s}\right)$ is computed by $T_{s}=N_{f} \tau \mathrm{msec}$. It should be noted that only the longest stimulus duration used is different for the two subjects: $1,829 \mathrm{msec}$ for Subject R.O. and, to avoid monotonous sessions, $1,371 \mathrm{msec}$ for Subject P.W.

\section{Results}

Discrimination thresholds $W$ for angular velocity $\Omega_{\text {ref }}=$ $80 \mathrm{deg} / \mathrm{sec}$ are plotted in Figure 2 as a function of frame time $(\tau=14 \ldots 114 \mathrm{msec})$, with the stimulus duration $T_{s}=N_{f} \tau$ as a parameter (with $N_{f}=2 \ldots 64$ ). In general, for a fixed frame time $\tau$, thresholds $W$ decrease monotonically as the number of frames $N_{f}$-and thus the stimulus duration $T_{s}$-is increased. Whereas the decrease of thresholds is dramatic if we go from a $57-\mathrm{msec}$ to a 114msec stimulus duration, the curves are bunched close together for stimulus durations $T_{s}>229 \mathrm{msec}$. For these stimulus durations $\left(T_{s}>229 \mathrm{msec}\right)$, thresholds $W$ are approximately constant $(W=6 \ldots 8 \%)$ for frame times $\tau$ between $14 \mathrm{msec}$ and $57 \mathrm{msec}$. However, if the frame time exceeds $57 \mathrm{msec}$, motion perception breaks down and thresholds increase strongly for all stimulus durations. For example, if we take a large stimulus duration, $T_{s}=$
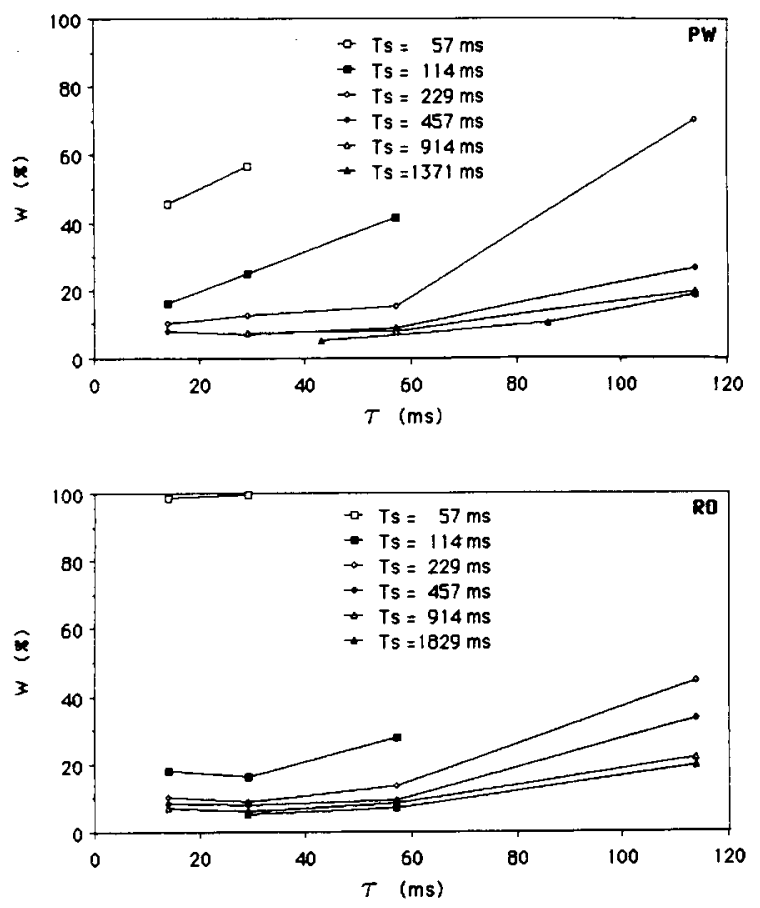

Figure 2. Discrimination thresholds $W$ for angular velocity are plotted as a function of frame time $(\tau)$ for several stimulus durations $T$. (Parameters: $\Omega_{\mathrm{ref}}=80 \mathrm{deg} / \mathrm{sec}, N_{d}=8, \rho_{0}=3 \mathrm{~cm}, D=100 \mathrm{~cm}$, $\epsilon=\mathbf{0 .})$
$914 \mathrm{msec}$, thresholds increase from $\simeq 7 \%$ for $\tau \leq 57$ msec to $\simeq 20 \%$ for $\tau=114 \mathrm{msec}$, for both subjects.

It should be noted that although thresholds are closer together for stimulus durations over $229 \mathrm{msec}$ and frame times less than $57 \mathrm{msec}$, thresholds still decrease slowly, but systematically, to thresholds $W=5.2 \%$ at $T_{s}=$ $1,371 \mathrm{msec}$, for Subject P.W. and $W=5.3 \%$ at $T_{s}=$ $1,829 \mathrm{msec}$, for Subject R.O.

The standard deviation of the threshold distribution is $\simeq 10 \%$ of the threshold for all data points.

\section{Discussion}

For a range of temporal parameters, marked by a frame time, $\tau \leq 57 \mathrm{msec}$, and a stimulus duration, $T_{s} \geq$ $229 \mathrm{msec}$, the psychophysical performance in this discrimination task is almost constant. These boundaries reflect the temporal characteristics of the angular velocity estimation mechanism at an angular velocity of $80 \mathrm{deg} / \mathrm{sec}$. Temporal integration can improve signal-to-noise ratios and can explain the decrease of the discrimination thresholds as $T_{s}$ increases. The minimum stimulus duration $T_{s} \simeq 229 \mathrm{msec}$ for optimal performance reveals the temporal integration time for the estimation of angular velocity. Integration times of the same order for linear velocity perception were reported by McKee and Welch $\left(1985 ; T_{s} \simeq 100 \mathrm{msec}\right)$ and by De Bruyn and Orban (1988; $T_{s} \simeq 200 \mathrm{msec}$ ). The frame time at which velocity perception starts to break down $(\tau=57 \mathrm{msec})$ can be associated with the delay time between two spatially separated inputs connected to a spatiotemporal correlator (Reichardt, 1987). Similar psychophysical estimations of the delay time $(\simeq 63 \mathrm{msec})$ at a linear retinal velocity of $\simeq 2.4 \mathrm{deg} / \mathrm{sec}$ were reported by van Doorn and Koenderink (1982a).

If motion perception breaks down at large frame times, positional information is left as the discrimination cue. For frame time $\tau=114 \mathrm{msec}$, for which motion perception is not smooth, thresholds exceed $18 \%$, even for a large number of frames $\left(N_{f}=32\right)$. At smaller $\tau$, for which motion perception is smooth, thresholds decrease markedly to $W=6 \ldots 8 \%$. This finding indicates that, at small $\tau$, velocity information is the primary cue for this discrimination task. Previously, Lappin and Bell (1976) and McKee (1981) showed that velocity discrimination is dependent neither on estimates of the total distance traversed by a target nor on the total target duration.

The reduction of the discrimination threshold (e.g., a factor 2.7, 2.1 at $\tau=57 \mathrm{msec}$ for Subjects P.W. and R.O., respectively) by doubling the number of frames from two to four (and thus $T_{s}$ from 114 to $229 \mathrm{msec}$ ) cannot entirely be understood by an improvement (by averaging the velocity estimates from different possible matches between consecutive frames) of signal-to-noise ratios alone. If angular velocity estimation is based on matches between pairs of consecutive frames only, a decrease in thresholds would be expected of a factor maximally $\sqrt{3}$ because a four-frame presentation contains three pairs of frames. A possible explanation for small frame times is that 
matches between noncontiguous frames (and thus longer time intervals) occur. However, the probability of these matches over an angle, $m \Omega_{\text {ref }} \tau$, and time, $m \tau$, is small for large $\tau$, where $m$ is an integer larger than 1 (Werkhoven \& Koenderink, 1989).

\section{Conclusion}

The temporal characteristics (temporal integration and delay time), as shown in this experiment, are very similar to those found for linear motion detection. Near optimal thresholds $[W=7.6 \%(8.0 \%)]$ are found at $\tau=$ $43 \mathrm{msec}$ and $N_{f}=8$, which allows experiments that are efficient with respect to time consumption.

\section{EXPERIMENT 2}

\section{Textural Characteristics}

It is generally known that the perceived angular velocity of a revolving pattern can be strongly affected by its spatial structure. For example, both an increase in the number of dots filling up a pattern and a decrease in their size can increase the perceived angular velocity (Walker, 1975). Moreover, Campbell showed that perceived angular velocity was dramatically slowed down or even stopped for high spatial frequency gratings (Campbell, 1979). In addition, neurophysiological experiments (Saito et al., 1986) revealed neurons in the dorsal part of the middle temporal area (MT) of the Macaque monkey that responded selectively to a frontoparallel rotation of textured patterns but not to a rotation of nontextured simple patterns such as rectangular bars. These experiments strongly suggest that perceived angular velocity can be affected by textural aspects. However, it is not known yet to what extent discrimination of angular velocity is affected by textural aspects.

\footnotetext{
Method

In this experiment, we examined the dependence of psychophysical performance for angular velocity discrimination on the number of dots of the annulus $\left(N_{d}\right)$ and their arrangement (e.g., the scatter $\epsilon$ in individual eccentricity). As described in the General Method section, the annulus consisted of $N_{d}$ dots with positions $\left(\rho_{i}, \phi_{i}\right)$ $\left(i=1 \ldots N_{d}\right)$, where the eccentricity, $\rho_{i}$, was chosen randomly within an interval $\left[(1-\epsilon) \rho_{0} \ldots(1+\epsilon) \rho_{0}\right]$. This annulus was rigidly rotated. It should be noted that, for every new trial, new scattered positions were assigned to the dots. We varied $N_{d}$ and $\epsilon$, whereas parameters $\rho_{0}, \Omega_{\text {ref }}, \tau$, and $N_{f}$ were kept constant (see General Method section).

In the following sections, the discrimination thresholds for an gular velocity as a function of $N_{d}$ at constant eccentricity $(\mathrm{c}=\mathbf{0})$ are noted as $W_{c}\left(N_{d}\right)$ and the thresholds at scattered eccentricity $(\epsilon=0.5)$ are noted as $W_{s}\left(N_{d}\right)$.
}

\section{Results}

In Figure 3, discrimination thresholds are plotted as a function of $N_{d}\left(N_{d}=1 \ldots 32\right)$ for two values of $\epsilon: \epsilon=0$ and $\epsilon=0.5$. From Figure 3, it is very clear that scattering the eccentricities of the individual dots of the annulus strongly affects the discrimination of angular velocity;
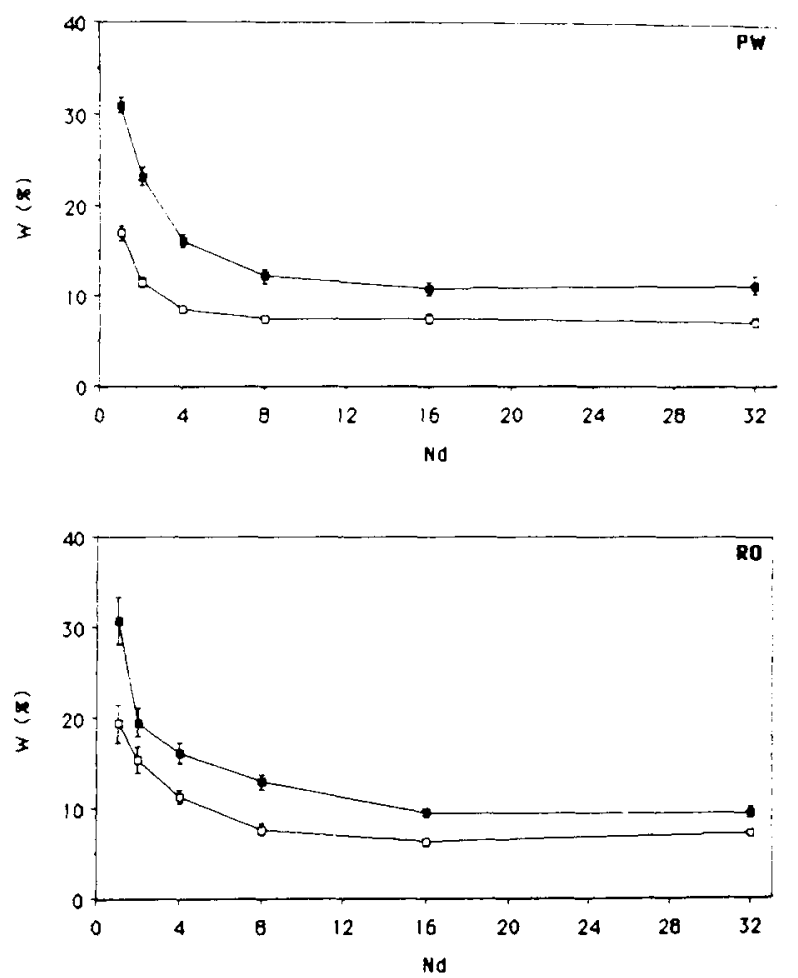

Figure 3. Discrimination thresholds $W$ for angular velocity are plotted as a function of the number of dots $\left(N_{d}\right)$ for unscattered eccentricities $\rho_{i}(\epsilon=0$ : open square) and scattered eccentricities of the dots for ( $\epsilon=0.5$ : solid square). (Parameters: $0_{\text {rof }}=80 \mathrm{dez} / \mathrm{sec}$, $\tau=43 \mathrm{msec}, N_{f}=8, \rho_{0}=3 \mathrm{~cm}, D=100 \mathrm{~cm}$.)

thresholds $W_{s}\left(N_{d}\right)$ are systematically higher than the thresholds $W_{c}\left(N_{d}\right)$ for dots arranged on a circle.

As a function of $N_{d}$, both curves in Figure 3 may be described as consisting of two parts: In the first part, thresholds decrease monotonically as the number of dots is increased from $N_{d}=1$ up to $N_{d}=8$. In the second part, for $N_{d}>8$, thresholds level off at $W_{c} \approx 7 \%$ [7\%] for $\epsilon=0$ and $W_{s}=11 \%[9 \%]$ for $\epsilon=0.5$.

\section{Hypothesis}

The dependence of discrimination thresholds on both $N_{d}$ and $\epsilon$ for the first part of the curve $\left(N_{d}=1 \ldots 8\right)$ can be understood by assuming that the average local linear velocity of the individual dots is used as the cue for the discrimination task.

First, it will be of interest to focus on the thresholds for one dot $\left(N_{d}=1\right)$. The angular velocity discrimination threshold for $N_{d}=1$ increases from $W_{c}(1)=17 \%$ [19\%] for a constant eccentricity to $W_{s}(1)=31 \%$ [31\%] when the eccentricity of the dot is scattered. At $\epsilon=0.5$, the standard-deviation of the eccentricity distribution $\sigma_{\rho}$ is: $\sigma_{\rho}=\rho_{0} / 2 \sqrt{3}$. Hence, $\sigma_{\rho} / \rho_{0} \simeq 29 \%$. Furthermore, let us take the threshold $W_{c}(1)(\epsilon=0)$ as the standard deviation of the estimation of the local linear velocity of one dot. If angular velocity estimates are mediated by local 
linear velocity estimates, the angular velocity estimates are affected by $\sigma_{\rho}$ and $W_{c}(1)$ independently, which predicts a threshold of $W_{s}(1)=\sqrt{\sigma_{o}^{2}+W_{c}(1)^{2}} \simeq 33 \%$ [35\%] for $\epsilon=0.5$. This is in close agreement with our finding: $W_{s}(1)=31 \%[31 \%]$. This observation suggests strongly that angular velocity estimates are indeed mediated by local linear velocity estimates. We conclude that angular velocity cannot be assessed directly by normalizing local linear velocities, $v_{i}$, with respect to their eccentricity, $\rho_{i}$. The perceived angular velocity, $\Omega$, of a dot seems to depend linearly on the eccentricity, $\rho_{i}$, of its trajectory.

To go ahead, in order to map the dependence of $W$ on the number of dots $N_{d}$, we hypothesize that angular velocity, $\Omega$, is not directly accessible to the visual system, but that instead the average, $\bar{v}$, of linear velocities, $v_{i}$, of the $N_{d}$ individual dots of the annulus is used as a cue for the discrimination task. For a certain angular velocity $(\Omega)$ and viewing distance $(D)$, the local linear velocity $\left(v_{i}\right)$ of an individual dot, $i$, is uniquely related to its eccentricity $\left(\rho_{i}\right)$. Since $\rho_{i} \ll D$, the average local velocity amplitude $(\bar{v})$ of the dots of a revolving annulus is:

$$
\bar{v} \approx \frac{1}{N_{d}} \frac{\pi \Omega}{180 D} \sum_{i=1}^{N_{d}} \rho_{i}(\mathrm{deg} / \mathrm{sec}) .
$$

Suppose that $\bar{v}$ is used as a cue for the angular velocity discrimination task. If $N_{d}$ dots are present in a pattern, the standard deviation of the measured average velocity, due to the scatter and the error in velocity estimates, is reduced with a factor, $\sqrt{N_{d}}$, with respect to the standard deviation of the velocity estimation of one dot, by averaging the local linear velocity estimates, $v_{i}$, of the $N_{d}$ individual dots. This would predict the relationship:

$$
W_{s}\left(N_{d}\right)=\frac{W_{s}(1)}{\sqrt{N_{d}}} \text { and } W_{c}\left(N_{d}\right)=\frac{W_{c}(1)}{\sqrt{N_{d}}} \text {. }
$$

The two curves should be related by

$$
W_{s}^{2}\left(N_{d}\right)=\frac{\sigma_{\rho}^{2}}{N_{d}}+W_{c}^{2}\left(N_{d}\right) .
$$

To check this relationship, we want to fit the function

$$
W\left(N_{d}\right)=\frac{\gamma}{N_{d}^{\alpha}}
$$

to the measured thresholds $W$ as a function of $N_{d}$ for both $\epsilon=0$ and $\epsilon=0.5$ for the first part of the curves, that is $N_{d}=1 \ldots 8$. We can rewrite Equation 5 as a linear relation:

$$
\ln W\left(N_{d}\right)=\ln \gamma-\alpha \ln N_{d} .
$$

Therefore, we performed a linear regression by a least square fit of the data points in double logarithmic coordinates for $N_{d}=1,2,4$, and 8 . As a measure of the goodness of fit, we used the regression coefficient, $r^{2}$, and the standard deviation of regression, $\chi^{2}$. The fit results for $\gamma$ and $\alpha$, as well as $r^{2}$ and $\chi^{2}$, are given in Table 2 for $\epsilon=0$ and $\epsilon=0.5$ for both subjects.
Table 2

Results of the Fit Procedure

\begin{tabular}{cccccc}
\hline Subject & $\epsilon$ & $\gamma($ \% $)$ & $\alpha$ & $\chi^{2}$ & $r^{2}$ \\
\hline P.W. & 0.0 & $16.8 \pm 0.7$ & $0.44 \pm 0.05$ & 9.0 & 0.87 \\
P.W. & 0.5 & $31.1 \pm 0.3$ & $0.46 \pm 0.02$ & 0.7 & 0.98 \\
R.O. & 0.0 & $20.5 \pm 1.2$ & $0.45 \pm 0.04$ & 0.9 & 0.87 \\
R.O. & 0.5 & $28.4 \pm 2.3$ & $0.39 \pm 0.06$ & 2.8 & 0.98 \\
\hline
\end{tabular}

The estimated exponent $\alpha$ is slightly, but significantly, smaller than the hypothesized value $\alpha=0.5$, for all cases. The calculated values for $\gamma$ for $\epsilon=0$ and $\epsilon=0.5$ are close to the values $W_{c}(1)$ and $W_{s}(1)$ and yield a predicted value of $26.2 \pm 0.6 \%[20 \pm 3.6 \%]$ for $\sigma_{\rho}$, which approaches the real value of $29 \%$. The high value $\chi^{2}=9.0$ for Subject P.W. and $\epsilon=0$ is due to the fact that in this case thresholds already start to level off at $N_{d}=8$. In this case, an improvement of the fit can be obtained by fitting the data points for only $N_{d}=1,2$, 4, yielding: $\alpha=0.50 \pm 0.03, \gamma=16.9 \pm 0.34 \%, \chi^{2}=$ 0.65 , and $r^{2}=1.00$. The $\chi^{2}$ values indicate that our data bear out the hypothesis reasonably well for a small number of dots.

\section{Discussion}

A question that still remains is why discrimination thresholds level off for $N_{d}>8$. One possible explanation is that the averaging of local linear velocities takes place only over a limited number of dots $\left(N_{d}=8\right)$. This would predict two different levels for $\epsilon=0$ and $\epsilon=0.5$. Another explanation is that for a certain number of dots, the error in the average velocity estimation becomes smaller than a noise level further on in the processing path. However, this would predict that the levels are the same for larger $N_{d}$. The observed constant difference in level thresholds supports the view that only a limited number of dots contributes to the averaging process.

Why are the discrimination thresholds, $W_{c}$, for the angular velocity of one dot $\left[W_{c}(1)=17.0 \%[19.4 \%]\right]$ so high? If eccentricity is not scattered $(\epsilon=0)$, and $\Omega_{\text {ref }}=80 \mathrm{deg} / \mathrm{sec}$, the instantaneous retinal velocity of the dot is $2.4 \mathrm{deg} / \mathrm{sec}$. This local retinal velocity could be used to discriminate angular velocity, because eccentricity is constant. Although they used a different target (a narrow line), McKee (1981) and Orban, Van Calenbergh, De Bruyn, and Maes (1985) reported velocity discrimination thresholds, $\sigma_{v}$, as low as $5 \%$ at such retinal velocities in the fovea. We examined three independent effects that can contribute to these discrimination thresholds, $W$. First, the sign of the angular velocity was varied randomly in our experiment to avoid motion aftereffects. This could cause an extra noise, $\sigma_{\text {sign }}$, in the decision stage of the discrimination task. However, control experiments for which the sign was kept constant showed that the thresholds were not affected by random signs of angular velocity. Second, the angle at which the dot started its trajectory was varied randomly between 0 and $2 \pi$. This random location of presentation could cause an uncertainty 
$\sigma_{\text {loc }}$ in the discrimination task. In a control experiment $(\epsilon=0)$, in which the dot always started its trajectory at the same point on the screen for both reference and test annulus, a decrease of the threshold was found to $11.8 \pm 0.5 \%$ [ $14.8 \pm 0.4 \%]$. Third, in our experiment the dot was moving at an eccentricity of $1.72^{\circ}$, instead of a foveal presentation, which can introduce an error $\sigma_{\mathrm{ecc}}$. Therefore, we carried out a control experiment in which both the location of presentation and the sign of rotation were kept constant as in the previous experiment. However, the subject was allowed to fixate on the location of the revolving dots. For these conditions, which are almost identical to the foveal presentation of a translating dot, thresholds were found of $10.2 \pm 0.4 \%$ [11.8 $\pm 0.9 \%$ ].

The errors $\sigma_{\nu}, \sigma_{\text {sign }}, \sigma_{\text {loc }}$, and $\sigma_{\text {ecc }}$ are assumed to be uncorrelated and to contribute independently to the final velocity threshold $W$ :

$$
W^{2}=\sigma_{v}^{2}+\sigma_{\text {sign }}^{2}+\sigma_{\text {loc }}^{2}+\sigma_{\text {ecc }}^{2}+\sigma_{?}^{2},
$$

where $\sigma$ ? gives an unexplained contribution to $W$. We can now estimate the independent contributions with the results of the control experiments: $\sigma_{\text {sign }}^{2} \simeq 0 \%, \sigma_{\text {loc }}^{2} \simeq$ $12 \%$ [13\%], and $\sigma_{\text {ecc }}^{2} \simeq 6 \%$ [9\%]. It should be noted that McKee used a somewhat different criterion (75\% correct responses) than we did $(84 \%)$. Therefore, the minimum thresholds of $5 \%$ found by McKee should be multiplied by 1.48 for comparison with our thresholds, yielding $\sigma_{v}=7.4 \%$. Thus, an error $\left[\sigma_{?} \simeq 7 \%[9 \%]\right]$ remains unexplained. However, we did not check the effects of a curved versus a linear trajectory or the effects of specific target shapes (narrow line versus dot).

To the best of our knowledge, the discrimination of angular velocity has been examined systematically in only a few previous experiments (Brandalise \& Gottsdanker, 1959; Kaiser, 1990). Brandalise and Gottsdanker (1959) had subjects adjust the angular velocity of a continuous revolving black disk with one white dot at $2.6^{\circ}$ eccentricity, such that the perceived angular velocity of the dot was equal to the angular velocity of a dot on a reference disk that was presented simultaneously. For a wide range of angular velocities $\left(\Omega_{\mathrm{ref}}=60 \ldots 540 \mathrm{deg} / \mathrm{sec}\right)$, they found thresholds varying nonsystematically between $5.9 \%$ and $9.5 \%$. These thresholds are lower than the thresholds found in our experiment. However, for a simultaneous presentation with an unlimited stimulus duration (as used in their experiment) it is possible to use the phase relations of the two revolving disks as a cue, which is not possible in our experiment.

Kaiser (1990) investigated observers' abilities at discriminating the rotational velocities of two simultaneously viewed polyhedral objects. Thresholds for angular velocity discrimination were approximately $10 \%$. Although we used a different paradigm and stimulus (dot patterns), we found similar results.

\section{Conclusion}

The hypothesis, that the average of local linear velocity estimates of the individual dots of the annulus is used as a cue for angular velocity estimation, is supported by the results of this experiment. The hypothesis suggests that perceived angular velocity is affected by the distribution of the dots, that is, the average eccentricity.

\section{EXPERIMENT 3 Spatial Scale}

If we assume that mechanisms tuned to rotary motion operate at a certain spatial scale (certain receptive field size), one might expect changes in performance when changing the spatial scale of the stimulus. For example, if the perception of rotary motion would be mediated by neurons similar to the neurons found by Saito et al. (1986) in the dorsal part of the MT of Macaque monkeys, one would expect the sensitivity diameter of these neurons (20 ... $30^{\circ}$ ) to be the optimal stimulus size. Julesz and Hesse (1970) showed in a psychophysical experiment that regions of a textured pattern composed of thousands of small elements could not easily be discriminated solely on the basis of differences in the direction of rotation of the elements. However, although with a completely different psychophysical paradigm, Regan and Beverley (1985) demonstrated the existence of visual mechanisms tuned to rotary motion with a stimulus size of $1^{\circ}$. If the mechanisms involved in the experiments referred to above (addressing directional information) are also involved in the estimation of angular velocity amplitudes, one may expect thresholds for angular velocity discrimination to vary with the scale of presentation.

In contrast, if our hypothesis that angular velocity discrimination is based on linear velocity estimates is true, we expect that thresholds are invariant for a wide range of spatial scales of the stimulus. The boundaries of this range would be determined by the minimum and maximum linear velocities that can be optimally discriminated.

To examine the dependence of angular velocity thresholds on spatial scale, we varied spatial scale in this experiment.

\section{Method}

Thresholds for an annulus composed of 8 dots with equal eccentricities $(\epsilon=0)$ were measured for one angular velocity $\left(\Omega_{\text {ref }}=\right.$ $80 \mathrm{deg} / \mathrm{sec}$ ) as a function of the viewing distance. The temporal parameters were $N_{f}=8$ and $\tau=43 \mathrm{msec}$. By varying the viewing distance $(D)$ between 7 and $1,200 \mathrm{~cm}$, the retinal eccentricity of the dots $\left[E_{0}=\arctan \left(\rho_{0} / D\right)\right]$ varied between 8.4 arcmin and $40.6^{\circ}$ of visual angle. The usual dot (size $0.17 \mathrm{~cm}$ ) became too small to be resolved at large viewing distances (e.g., the retinal dot size is 0.49 arcmin at a viewing distance $D=1,200 \mathrm{~cm}$ ). To widen the range of viewing distances at which the dots can be resolved, we used a bigger dot (size $0.31 \mathrm{~cm}$, which is 0.89 arcmin at $D=$ $1,200 \mathrm{~cm}$ ) in this experiment. Furthermore, for practical reasons, we preferred to use a dot eccentricity of $\rho_{0}=6 \mathrm{~cm}$ at a viewing distance $D=7 \mathrm{~cm}$, instead of a dot eccentricity $\rho_{0}=3 \mathrm{~cm}$ at a viewing distance $D=3.5 \mathrm{~cm}$, in order to obtain the largest eccentricity $\left(E_{0}=40.6^{\circ}\right)$.

\section{Results}

Weber fractions $(W)$ for angular velocity are plotted as a function of retinal dot eccentricity $\left(E_{0}\right)$ in Figure 4. It appears that Weber's law of the invariance of relative 

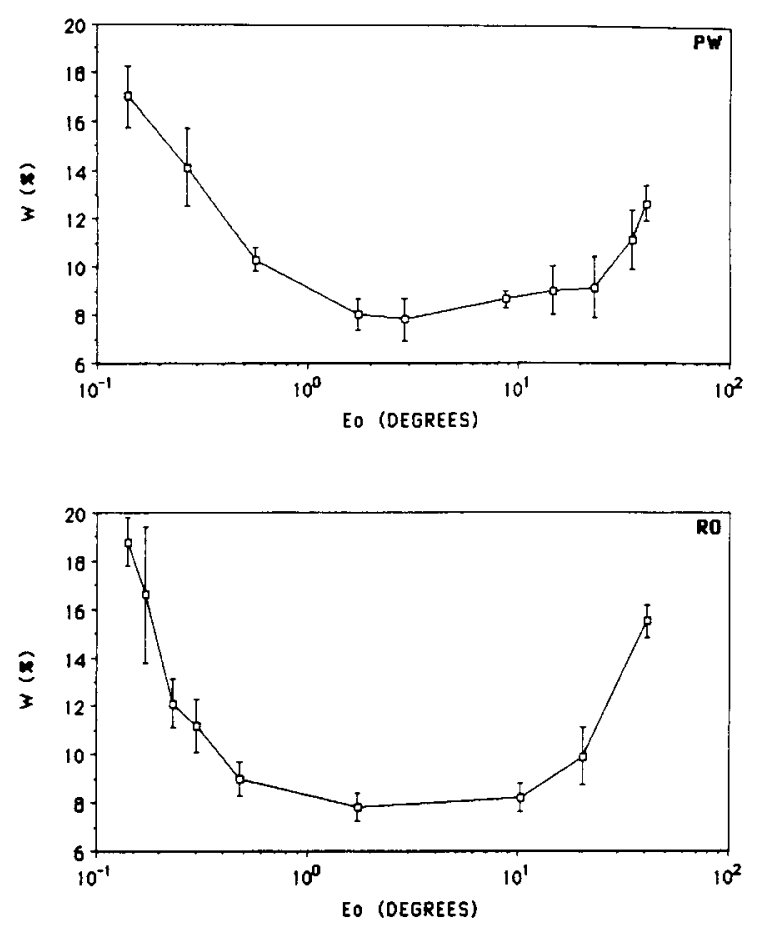

Figure 4. Discrimination thresholds $W$ for angular velocity are plotted as a function of the eccentricity $E_{0}$ of the dots. (Parameters: $\mathrm{Q}_{\mathrm{ref}}=80 \mathrm{deg} / \mathrm{sec}, \tau=43 \mathrm{msec}, N_{f}=8, N_{d}=8, \epsilon=0$.)

discrimination thresholds to spatial scale is applicable for a range of retinal eccentricities from $E_{0} \simeq 0.5^{\circ}$ up to at least $E_{0}=23.2^{\circ}$ of visual angle. Discrimination thresholds are approximately constant $(W=8 \ldots 10 \%)$ over this range. Outside this range, the thresholds increase gradually to $17 \%$ [19\%] for smaller eccentricity $\left(E_{0}=\right.$ 8.4 arcmin) and to $13 \%$ [15\%] for larger eccentricity $\left(E_{0}=40.6^{\circ}\right)$. The differences in thresholds for dot size $0.88 \mathrm{arcmin}$ (this section) and dot size $0.49 \mathrm{arcmin}$ (see data of Experiments 1 and 2) at a viewing distance of $D=$ $100 \mathrm{~cm}\left(E_{0}=1.72^{\circ}\right)$ are small.

\section{Discussion}

The results of this experiment did not reveal discontinuous changes or local minima of the thresholds at certain spatial scales, which could provide evidence for separate mechanisms tuned to rotary motion and operating at a specific spatial scale of the stimulus. Discrimination thresholds are approximately constant $(W=8 \ldots$ $10 \%$ ) over a wide range of retinal dot eccentricities of $E_{0} \simeq 0.5^{\circ}$ up to at least $E_{0}=23^{\circ}$. In terms of the retinal linear velocities, $v_{i}$, of individual dots, this range is marked by a lower boundary for the retinal velocity of $0.8 \mathrm{deg} / \mathrm{sec}$ and an upper boundary of $39 \mathrm{deg} / \mathrm{sec}$. These boundaries agree reasonably well with the range (between 4 and $64 \mathrm{deg} / \mathrm{sec}$ ) found by De Bruyn and Orban (1988) for linear motion of random dot patterns and the lower boundary of $2 \mathrm{deg} / \mathrm{sec}$ found by McKee (1981) for a moving narrow line.

\section{Conclusion}

The invariance of the thresholds for a wide range of spatial scales supports our hypothesis that the estimation of angular velocity is mediated by local linear velocity estimates of the individual dots.

\section{EXPERIMENT 4 \\ Weber Law for Angular Velocity Discrimination}

The existence of a Weber's law of the invariance of relative thresholds for velocity is well known (De Bruyn \& Orban, 1988; McKee, 1981). In this experiment, we attempt to determine if a similar behavior for the discrimination of angular velocity can be observed as a function of angular velocity amplitude.

\section{Method}

From Experiment 1, we know that the discrimination thresholds for an angular velocity of $80 \mathrm{deg} / \mathrm{sec}$ are approximately the same for $N_{f}=8, \tau=43$ and $N_{f}=24, \tau=14$. For higher angular velocities, this is not the case. For larger angular velocities, the displacement of the dots from one frame to the next can even become larger than half of the separation between two neighboring dots, such that reversed motion perception occurs. A gradual increase of reversed motion perception probability can be expected as the displacement increases. For example, at $\tau=43 \mathrm{msec}$, subjects reported reversed motion perception due to aliasing at angular velocities exceeding $\approx 300 \mathrm{deg} / \mathrm{sec}$. At $\tau=14 \mathrm{msec}$, however, reversed motion was reported for angular velocities exceeding a much higher velocity of $850 \mathrm{deg} / \mathrm{sec}$ (Werkhoven \& Koenderink, 1989). For this reason, we used the frame time $\tau=14 \mathrm{msec}$ and $N_{f}=24$ in this experiment in order to be able to vary the angular velocity up to $320 \mathrm{deg} / \mathrm{sec}$ without aliasing effects.

\section{Results}

Discrimination thresholds as a function of angular velocity amplitude are plotted in Figure 5. For both subjects, thresholds are approximately constant for angular velocities $\Omega_{\text {ref }}$ between $80 \mathrm{deg} / \mathrm{sec}$ and at least $320 \mathrm{deg} / \mathrm{sec}$. For $\Omega_{\text {ref }}<80 \mathrm{deg} / \mathrm{sec}$, thresholds increase rapidly up to $W=23 \%$ for $\Omega_{\text {ref }}=10 \mathrm{deg} / \mathrm{sec}$.

\section{Discussion}

Regarding local retinal velocities, we can say that the ability to discriminate angular velocity decreases as soon as the instantaneous retinal linear velocities of the individual dots, $v_{i}$, become smaller than $2.4 \mathrm{deg} / \mathrm{sec}$. This is in accordance with the experiments of De Bruyn and Orban (1988), who showed that linear velocity discrimination displays a U-shaped dependence on velocity. A lower boundary of $\approx 4 \mathrm{deg} / \mathrm{sec}$ was found for the range of minimal thresholds. Based on the same U-shaped curves of De Bruyn and Orban (1988) for almost continuous motion, we expect that the ability to discriminate angular velocity breaks down at an angular velocity of approximately $2,130 \mathrm{deg} / \mathrm{sec}$ (i.e., $v_{i} \simeq 64 \mathrm{deg} / \mathrm{sec}$ ). Although the estimated cut-off angular velocity may be lower for stroboscopic presentations, we could not measure this high limit for angular velocity discrimination, due to the periodic spatial structure of the stimulus. We 

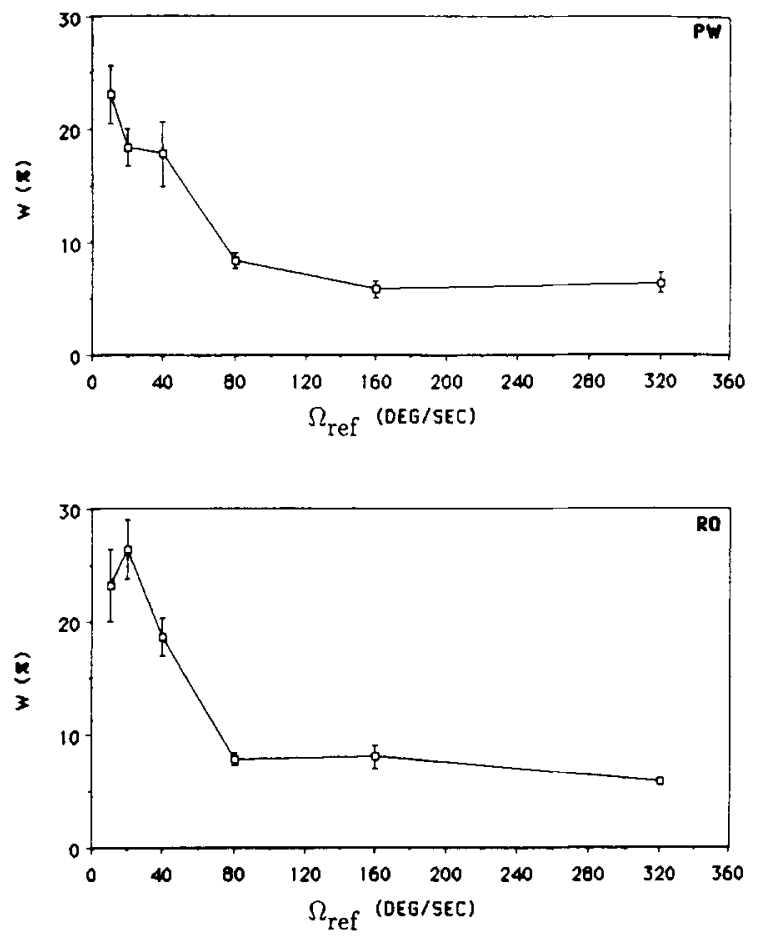

Figure 5. Discrimination thresholds $W$ for angular velocity are plotted as a function of angular velocity $D_{\text {ref }}$. (Parameters: $\tau=$ 14 msec, $N_{f}=24, N_{d}=8, p_{0}=3 \mathrm{~cm}, D=100 \mathrm{~cm}, \epsilon=0$.)

used a maximal angular velocity of $320 \mathrm{deg} / \mathrm{sec}$ where the retinal linear velocity, $v_{i}$, of the individual dots was $9.6 \mathrm{deg} / \mathrm{sec}$.

\section{GENERAL DISCUSSION}

Our experiments reveal the following characteristics of the psychophysical discrimination of angular velocity: (1) Temporal integration of the angular velocity information takes place over $\simeq 200$ msec. Integration times of the same order for linear velocity perception were reported by De Bruyn and Orban (1988). (2) The frame time at which angular velocity perception starts to break down $(57 \mathrm{msec})$ is consistent with temporal delay values for a spatiotemporal correlation model for the estimation of linear velocity, as reported by van Doorn and Koenderink (1982a). (3) Scattering the eccentricities of the dots affects the discrimination thresholds, as can be expected when the averaged local velocities of the individual dots appear to be used as a cue for the discrimination task. (4) Angular velocity discrimination displays a $U$-shaped dependence on the scale of the annulus, with minimal thresholds of 7\%. The limits for angular velocity discrimination seem to be determined by the limits for linear velocity discrimination, as reported by De Bruyn and Orban (1988).

All observations show a strong analogy between angular velocity and linear velocity discrimination. This supports our claim that the estimation of angular velocity in our experiments cannot be assessed directly but is the result of an average process of local linear velocity estimates or a weighted integration of the velocity field. Moreover, discrimination thresholds for angular velocity are not better than those produced by a weighted integration of the velocity field. Experiments on the discrimination of the direction of revolving random dot patterns, done by Bell and Lappin (1979), can complement our findings. Their results suggest that the perceptual process underlying the detection of coherent motion are the same, regardless of whether that motion is based upon the rotation or translation of the individual pattern elements.

The results of Experiment 2 indicated that the angular velocity estimation of a dot cannot be assessed but is based on linear velocity estimates and thus linearly related to the distance of the dot to the center of rotation. This suggests strongly that the perceived angular velocity of an object will depend on the spatial extension of the object. Moreover, if based on linear velocity estimates, the discrimination of angular velocity should be seriously affected by a superposition of translational motion (or other motion parallax components such as divergence and deformations). For the visibility of movement gradients, this was already shown by van Doorn and Koenderink (1982c). In this context, Nakayama (1985) mentioned three reasons for this, one of which is the Weber law for differential velocity detection. Additional experiments might provide evidence for these predictions.

Although, in our experiment, the average of local linear velocity amplitudes is used by subjects to discriminate angular velocity, it is not ruled out that additional information is processed in other situations. For example, adaptation experiments (Regan \& Beverley, 1985) show that the phase relationship between oscillating dots in a revolving pattern is processed and determines adaptation thresholds.

One last, but important, question that remains is whether or not higher order variables of velocity fields (such as rotation) can be assessed at a conscious level, as is done in our experiments. One might argue that special mechanisms have evolved to estimate higher order variables of velocity fields, with lower thresholds than are found in our experiment, but that this information is fed directly into higher order routines and is not explicit at a conscious level. Therefore, additional experiments on the human psychophysical performance in relation either to form perception or to ego motion, using motion parallax components, are necessary.

\section{REFERENCES}

ANSTIS, S. (1986). Recovering motion information from luminance. Vision Research, 26, 147-159.

BeLL, H. H., \& LAPPIN, J. S. (1979). The detection of rotation in randomdot patterns. Perception \& Psychophysics, 26, 415-417.

Brandalise, B. B., GotTsdanker, R. M. (1959). The difference threshold of the magnitude of visual velocity. Journal of Experimental Psychology, 57, 83-88.

Campbell, F. W. (1979). Stopped visual motion. Nature, 278, 192. De Bruyn, B., Orban, G. A. (1988). Human velocity and direction 
discrimination measured with random dot patterns. Vision Research, 28, 1323-1335.

Gibson, J., Olum, P., \& Rosenblatt, F. (1958). Parallax and perspective during aircraft landings. American Journal of Psychology, $68,372-385$.

GorDon, D. A. (1965). Static and dynamic visual fields in human space perception. Journal of the Optical Society of America, 55, 1296-1303.

Helmholtz, H. von (1925). Treatise on Physiological Optics. (J. P. Southall, Trans.), New York: Dover.

HofFMAN, D. D. (1982). Inferring local surface orientation from motion fields. Journal of the Optical Society of America, 72, 888-892.

HoRN, B. K. P., Schunck, B. G. (1981). Determining optical flow. Artificial Intelligence, 17, 185-203.

JUlesz, B., HESSE, R. I. (1970). Inability to perceive the direction of rotation of line segments. Nature, 225, 243-244.

KaISER, M. K. (1990). Angular velocity discrimination. Perception \& Psychophysics, 47, 149-156.

KoENDERINK, J. J., \&AN DOORN, A. J. (1975). Invariant properties of the motion parallax field due to the movement of rigid bodies relative to an observer. Optica Acta, 22,773-791

KoENDERINK, J. J., \& VAN DOORN, A. J. (1976). Local structure of movement parallax of the plane. Journal of the Optical Society of America, 66, 717-723.

Koenderink, J. J., \& VAN DoORn, A. J. (1986). Depth and shape from differential perspective in the presence of bending deformations. Journal of the Optical Society of America A, 3, 242-249.

LAPPIN, J. S., \& BELL, H. H. (1976). The detection of coherence in moving random-dot patterns. Vision Research, 16, 161-168.

Longuet-Higgens, H., \& PrazdNY, K. (1980). The interpretation of a moving retinal image. Proceedings of the Royal Society London $B$, 208, 385-397.

$\mathrm{MACH}_{\mathrm{A}}$ E. (1962). The analysis of sensations. New York: Dover. (Original work published 1886)

Marr, D., Ullman, S. (1981). Directional selectivity and its use in early visual processing. Proceedings of the Royal Society London B, 211, $151-180$.

MCKeE, S. P. (1981). A local mechanism for differential velocity detection. Vision Research, 21, 491-500.

MCKEE, S. P., \& WELCH, L. (1985). Sequential recruitment in the discrimination of velocity. Journal of the Optical Society of America A, 2, 243-251.

Moulden, B., BegG, H. (1986). Some tests of the Marr-Ullmann model of movement detection. Perception, 15, 139-155.

Nakayama, K. (1985a). Biological image motion processing: A review Vision Research, 25, 625-660.

NAKA YAMA, K. (1985b). Higher order derivatives of the optical velocity vector field: limitations imposed by biological hardware. In $\mathrm{D}$. Ingle, M. Jeannerod, \& D. Lee (Eds.), Brain mechanisms and spatial vision. The Netherlands: Martinus Nijhoff.

Nakayama, K., Loomis, J. M. (1974). Optical velocity patterns, velocity-sensitive neurons, and space perception: A hypothesis. Perception, 3, 63-80.

Orban, G. A., Van Calenbergh, F., De Bruyn, B., \& Maes, H.
(1985). Velocity discrimination in central and peripheral visual field. Journal of the Optical Society of America A, 2, 1836-1847.

REGAN, D. (1986). Visual processing of four kinds of relative motion. Vision Research, 26, 127-145.

REgAN, D., \& BeVERLEY, K. I. (1985). Visual responses to vorticity and the neural analysis of optic flow. Journal of the Optical Society of America A, 2, 280-283.

REICHARDT, W. (1987). Evaluation of optical motion information by movement detectors. Joumal of Comparative Physiology A, 161, 533-547.

RIEGER, J. H. (1983). Information in optical flows induced by curved paths of observation. Journal of the Optical Society of America, 73, 339-344.

Rjemersma, J. B. J. (1981). Visual control during straight road driving. Acta Psychologica, 48, 215-225.

Rogers, B., \& Graham, M. (1979). Motion parallax as an independent cue for depth perception. Perception, 8, 125-134.

Saito, H., Yukie, M., Tanaka, K., Hikosaka, K., Fukada, Y., \& IWAI, E. (1986). Integration of direction signals of image motion in the superior temporal sulcus of the macaque monkey. Journal of Neuroscience, 6, 145-157.

Tanaka, K., Fukada, Y., \& SaIto, H. (1989). Underlying mechanisms of the responser specificity of expansion/contraction and rotation cells in the dorsal part of the medial superior temporal area of the Macaque monkey. Journal of Neurophysiology, 62, 642-656.

TANAKA, K., \& SAITo, H. (1989). Analysis of motion of the visual field by direction, expansion/contraction, and rotation cells in the dorsal part of the medial superior temporal area of the Macaque monkey. Journal of Neurophysiology, 62, 626-641.

van DoORn, A. J., \&oenderink, J. J. (1982a). Temporal properties of the visual detectability of moving spatial white noise. Experimental Brain Research, 45, 179-188.

vaN DOORN, A. J., KoENdERINK, J. J. (1982b). Spatial properties of the visual detectability of moving spatial white noise. Experimental Brain Research, 45, 189-195.

VAN DOORN, A. J., \& KOENDERINK, J. J. (1982c). Visibility of movement gradients. Biological Cybernetics, 44, 167-175.

van Santen, J. P. H., Sperling, G. (1985). Elaborated Reichardt Detectors. Joumal of the Optical Society of America A, 2, 300-321.

WALKER, J. T. (1975). Visual texture as a factor in the apparent velocity of objective motion and motion aftereffects. Perception \& Psychophysics, 18, 175-180.

Warren, W. H., Jr., Morris, M. W., \& Kalish, M. (1988). Perception of translational heading from optical flow. Joumal of Experimenial Psychology: Human Perception \& Performance, 14, 646-660.

Waxman, A. M., Kamar-Parsi, B, Subbarao, M. (1987). Closedform solutions to image flow equations. Proceedings of the First International Conference on Computer Vision (pp. 12-24). London.

WerkHoven, P., Koenderink, J. J. (1989). Reversed rotary motion perception. Unpublished manuscript.

(Manuscript received March 15, 1990;

revision accepted for publication September 4, 1990.) 